\title{
Subdural Hemorrhage Due to Acquired Von Willebrand Syndrome in a Patient With Polycythemia Vera
}

\author{
Khalid Shalaby ${ }^{1}$, Jussie Correia Lima ${ }^{2}$, Robert Szulawski ${ }^{3}$ \\ 1. Internal Medicine, University of Connecticut Health, Farmington, USA 2. Neurology, Hartford Hospital, Hartford, \\ USA 3. Internal Medicine, Hartford Hospital, Hartford, USA
}

Corresponding author: Khalid Shalaby, khalid.shalaby@gmail.com

\begin{abstract}
Polycythemia vera (PV) is a chronic myeloproliferative neoplasm associated with thrombosis. A 48-year-old female with PV presented with right eye pain following a low-impact head trauma. She consumed aspirin for analgesia and took preparations of Chinese herbs. CT head revealed right-sided subdural hematoma. She had reduced Von Willebrand activity to $26 \%$. Direct angiographic imaging showed an aneurysm arising from a right middle cerebral atery (MCA) branch. The patient was given 1-deamino-8-D-arginine vasopressin (DDAVP) prior to the craniotomy. Intra-operative examination revealed that the aneurysm-like structure was a small grape-like structure of the fibrinous part of the subdural membrane that had formed from the subdural hematoma. Acquired von Willebrand syndrome (AVWS) is an important risk factor for bleeding in PV. DDAVP may be useful to increase levels of Von Willebrand Factor (VWF) and decrease the risk of bleeding perioperatively. Exogenous substances such as ginseng should be investigated as possible contributors to bleeding tendency and discontinued.
\end{abstract}

Review began 06/30/2021 Review ended 07/18/2021 Published 07/25/2021

๑) Copyright 2021 Shalaby et al. This is an open access article distributed under the terms of the Creative Commons Attribution License CC-BY 4.0., which permits unrestricted use, distribution, and reproduction in any medium, provided the original author and source are credited.
Categories: Neurology, Neurosurgery, Hematology

Keywords: von willebrand diseases, myeloproliferative disorder, intracranial hemorrhage, chinese herbal drugs, platelet function test, ddavp

\section{Introduction}

Polycythemia vera (PV) is a chronic myeloproliferative neoplasm (MPN) characterized by an increase in hematocrit, RBC count, and hypercellularity of bone marrow [1]. PV affects one to two individuals per 100,000 person-years in the United States [2]. Symptoms include fevers, night sweats, weight loss, pruritis as well as microvascular complications manifesting as headache, dizziness, and numbness [1]. Macrothrombosis is one of the most common major complications of PV and occurs in $34 \%-39 \%$ of patients at the time of diagnosis [3]. Patients with low-risk PV (<60 years old and no history of cardiovascular events) are treated with serial phlebotomy with a target hematocrit $<45 \%$ and low dose aspirin for primary prophylaxis of thrombotic events $[4,5]$. Paradoxically, patients with PV may also have inhibited hemostasis and a high degree of clinical suspicion is needed to prevent potentially life-threatening complications. Here, we report a case of intracranial bleeding in PV due to acquired Von Willebrand syndrome (AVWS) with concomitant acquired platelet dysfunction due to aspirin and herbal remedies.

\section{Case Presentation}

A 48-year-old female with a past medical history of low-risk JAK2 positive PV presented to the emergency department with a chief complaint of right eye pain for eight days. Her pain started after she had an accidental low-impact trauma inflicted by her child. She described non-pulsatile, dull pain with increasing intensity overlying her right eye. She had no visual changes in her right eye and unchanged chronic left eye myopia. To attenuate the pain, the patient consumed 12 tablets of $81 \mathrm{mg}$ aspirin every day for three days at the onset of pain. She took preparations of ginseng and other unspecified Chinese herbs and prepared a herbal poultice to place over her right eye.

Her PV was diagnosed in China more than 15 years ago. She was compliant with therapeutic phlebotomies every two months for treatment of her PV, however recently she was not able to get to the clinic due to novel coronavirus restrictions. She was treated in the past with Hydroxyurea but was discontinued due to severe mucositis. Three years before presentation to our institution, she had been having heavy menstrual bleeding. Ristocetin-induced platelet aggregation assay showed decreased Von Willebrand factor (VWF) activity. Therefore, scheduled $81 \mathrm{mg}$ of aspirin daily was stopped. Abdominal ultrasound, performed for the indication of abdominal swelling, revealed splenomegaly with $19.4 \mathrm{~cm}$ spleen in maximal dimension as well as a fully distinct $2 \mathrm{~cm}$ splenule.

At the time of presentation to our institution, she had no skin or eyelid wounds, mucosal bleeding, menorrhagia, joint swelling, rash, bruises, or neurological deficits. She has no family history of coagulopathy. Her vital signs were within normal limits. Her neurological exam revealed normal extraocular 


\section{Cureus}

muscle movement, normal visual fields, decreased left eye visual acuity that remains unchanged from previous encounters. The rest of her neurological examination was unremarkable.

CT head without contrast was significant for right-sided subdural hematoma with a $6 \mathrm{~mm}$ right-to-left subfalcine shift and mass effect noted in the right ventricle without evidence of hydrocephalus (Figure $1 \mathrm{~A}$ ). CTA scan of the head and neck showed a 4-mm cortically based vascular malformation or suspected aneurysm observed within the right parieto-occipital region (Figure $1 B$ ). At the time of admission, her hemoglobin was $14.8 \mathrm{gm} / \mathrm{dL}$, hematocrit was 53.6\%, mean corpuscular volume was $68 \mathrm{fL}$, platelet count was $1,029,000 / \mu \mathrm{L}$, and white blood cell count was $24,800 / \mu \mathrm{L}$. Peripheral blood smear showed microcytosis, hypochromia, and polychromasia. Prothrombin time/international normalized ratio (PT/INR) and partial thromboplastin time (PTT) were within normal laboratory limits. VWF activity was reduced to $26 \%$. She had a normal factor VIII assay. Platelet mapping revealed no inhibition to arachidonic acid (AA), but significant inhibition to adenosine diphosphate (ADP) (60\% inhibited) was observed with a significant reduction in the mean amplitude of clot.
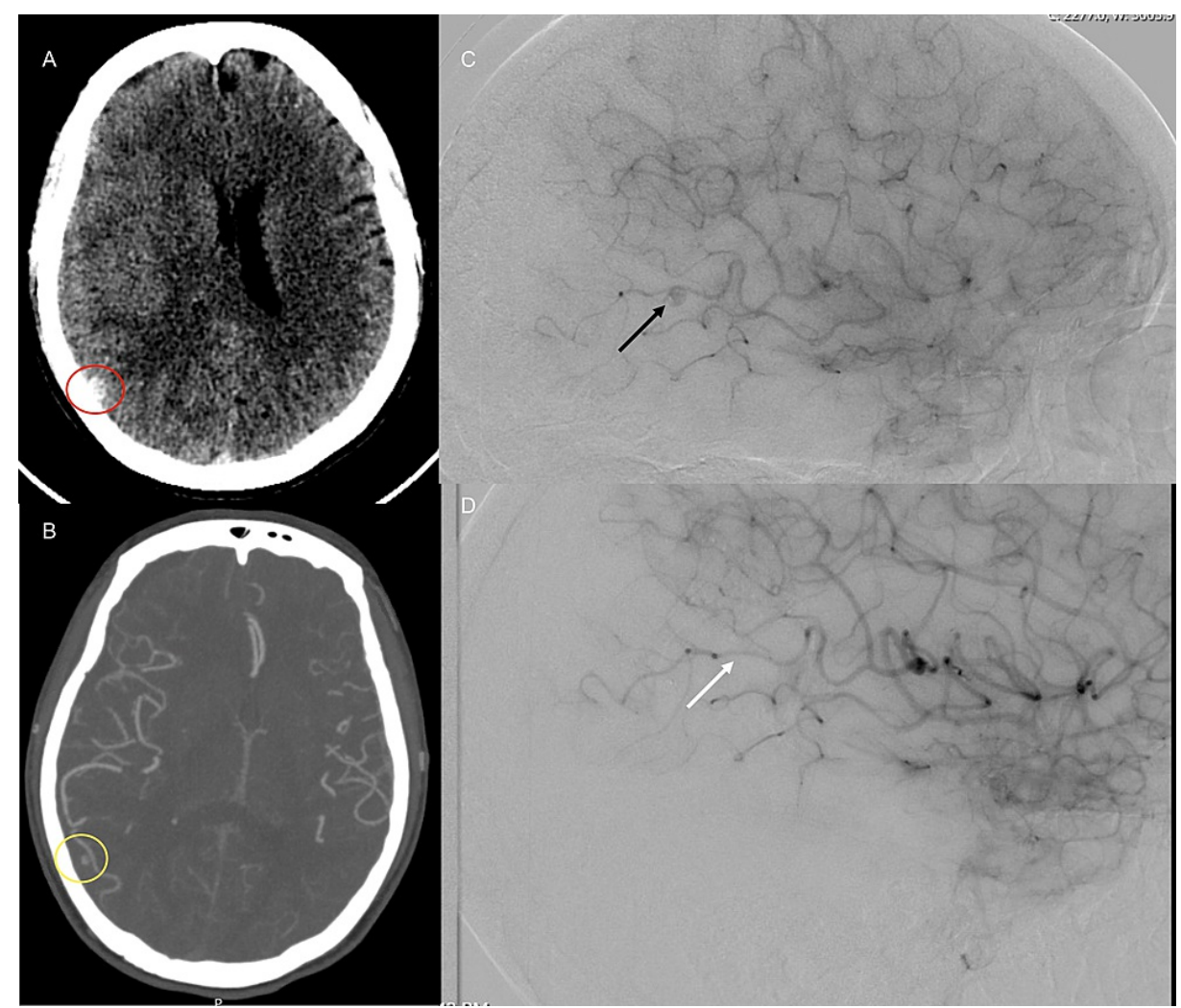

\section{FIGURE 1: Diagnostic imaging of the head}

(A) CT head without contrast: Red circle - Right-sided subdural hematoma with mild mass effect. (B) CTA head: Yellow circle - Aneurysm within the right parieto-occipital region. (C) Diagnostic cerebral angiogram: Black arrow - Inferolaterally projecting aneurysm arising from the mid aspect of an M4 right middle cerebral artery branch, specifically the angular artery measuring $3.6 \times 3.9 \mathrm{~mm}$. (D) Diagnostic cerebral angiogram (post-surgery): White Arrow - Aneurysm is no longer visualized status post craniotomy and evacuation of subdural hematoma.

Magnetic resonance angiography (MRA) revealed a possible saccular aneurysm, measuring up to $0.7 \mathrm{~cm}$, along the lateral aspect of the right parietal lobe associated with a distal branch of the right middle cerebral artery (MCA). Apparent clotted blood material surrounding this aneurysm was observed. Direct angiographic imaging of the right internal carotid artery was then performed which showed an inferolaterally projecting aneurysm arising from the mid aspect of M4 right MCA branch, specifically the angular artery. The aneurysm in question measured $3.6 \times 3.9 \mathrm{~mm}$. The neck of the aneurysm was relatively wide, measuring $3.2 \mathrm{~mm}$ (Figure 1C). The right anterior and remaining right middle cerebral artery branches were normal in appearance without significant stenosis or major branch vessel occlusion.

Neurosurgical intervention was recommended as the distal position of the aneurysm made endovascular access difficult and the wide-necked morphology of the aneurysm was not amenable to vascular intervention. The patient was given 1-deamino-8-D-arginine vasopressin (DDAVP) prior to the surgery to reduce the risk of bleeding. She underwent right craniotomy and intra-op examination revealed that directly underneath the dura, there was a very thick membrane of fibrinous material as well as hemorrhage. There 
was a large grape-like membrane that was attached to the pia-arachnoid. There was some bleeding noted from one of the pial arterial branches on the surface. This thickened grape-like membrane was then detached from the underside of the dura and sent off to pathology. Intraoperative microscopic evaluation of the angular artery of the middle cerebral artery revealed no evidence of an aneurysm. This aneurysm-like structure was in fact a small grape-like structure of the fibrinous part of the subdural membrane that had formed from the subdural hematoma that was being fed by a pial branch of the middle cerebral artery through a parasitized process. The pathological examination of the excised specimen was consistent with a subdural membrane showing organized hematoma with highly vascularized granulation tissue. In the post craniotomy diagnostic cerebral angiogram, the aneurysm was no longer visualized. (Figure 1D). The patient recovered well postoperatively with no clinical evidence of rebleeding. She was discharged home one day later.

\section{Discussion}

While the most common complication of PV is thrombotic events, patients with PV can rarely present with sequelae of bleeding. AVWS is an important risk factor for bleeding in PV patients [6,7] and occurs more commonly in patients with a platelets count of more than one million/dL [8]. In instances of abnormal bleeding with MPNs, it is advisable to test for disseminated intravascular coagulopathy and AVWS with VWF antigen and activity in acutely ill patients [8]. AVWS in MPN primarily results from direct adsorption of high molecular weight multimers of VWF to blood cells, in particular platelets [9]. Cytoreductive measures are reported to reduce bleeding and result in remission of AVWS [10]. Severe thrombocytosis (>1.5 million/dL) may warrant plateletpheresis [8].

DDAVP may be useful to increase levels of VWF and decrease the risk of bleeding perioperatively and in lifethreatening bleeding incidents [9]. One caveat to the use of DDAVP is that it is not always effective in the treatment of AVWS. This is attributed to the presence of immunoglobulin inhibitors of VWF, tachyphylaxis, or endothelial stores depletion [10,11]. In this case, we gave DDAVP to decrease the risk for bleeding during the surgery and the risk for recurrence of the subdural hematoma. If bleeding persists despite treatment with DDAVP, high dose factor VIII concentrate and intravenous immunoglobulins have been reported to be useful in controlling bleeding [9].

Exogenous substances should also be investigated as possible contributors to bleeding tendency and discontinued [9]. It is curious in this case that platelet mapping did not reveal any inhibition to AA despite the last aspirin use approximately five days before presentation. Marked attenuation of the ADP effect was found. We suspect that hypersplenism dramatically reduced the effective half-life of the aspirin-inhibited platelets leading to this unexpected effect [12]. Chinese herbs, including ginseng, are known to exert significant antiplatelet effects [13], and this was responsible for the potent attenuation of the ADP effect as the patient had continued taking herbals until presentation.

\section{Conclusions}

While many cases of bleeding in MPNOassociated AVWS are minor, this case illustrates that major complications of AVWS can manifest after minor trauma. Clinicians should always be aware of the potential for major bleeding tendency, especially when multiple pathways in hemostasis are suppressed. Cytoreduction is crucial in the prevention of AVWS in PV and essential thrombocythemia. DDAVP is used in the treatment of AVWS preoperatively and in life-threatening bleeding. Plateletpheresis, intravenous immunoglobulins, and factor VIII concentrate are important lines of therapy when DDAVP is ineffective.

\section{Additional Information \\ Disclosures}

Human subjects: Consent was obtained or waived by all participants in this study. Conflicts of interest: In compliance with the ICMJE uniform disclosure form, all authors declare the following: Payment/services info: All authors have declared that no financial support was received from any organization for the submitted work. Financial relationships: All authors have declared that they have no financial relationships at present or within the previous three years with any organizations that might have an interest in the submitted work. Other relationships: All authors have declared that there are no other relationships or activities that could appear to have influenced the submitted work.

\section{References}

1. Cuthbert D, Stein BL: Polycythemia vera-associated complications: Pathogenesis, clinical manifestations, and effects on outcomes. J Blood Med. 2019, 10:359-71. 10.2147/JBM.S189922

2. Shallis RM, Wang R, Davidoff A, Ma X, Podoltsev NA, Zeidan AM: Epidemiology of the classical myeloproliferative neoplasms: the four corners of an expansive and complex map. Blood Rev. 2020, 42:100706. 10.1016/j.blre.2020.100706

3. Tefferi A, Elliott M: Thrombosis in myeloproliferative disorders: prevalence, prognostic factors, and the role of leukocytes and JAK2V617F. Semin Thromb Hemost. 2007, 33:313-20. 10.1055/s-2007-976165

4. Marchioli R, Finazzi G, Specchia G, et al.: Cardiovascular events and intensity of treatment in polycythemia vera. N Engl J Med. 2013, 368:22-33. 10.1056/NEJMoa1208500 


\section{Cureus}

5. Landolfi R, Marchioli R, Kutti J, Gisslinger H, Tognoni G, Patrono C, Barbui T: Efficacy and safety of lowdose aspirin in polycythemia vera. N Engl J Med. 2004, 350:114-24. 10.1056/NEJMoa035572

6. Martin K: Risk factors for and management of MPN-associated bleeding and thrombosis . Curr Hematol Malig Rep. 2017, 12:389-96. 10.1007/s11899-017-0400-3

7. Kander EM, Raza S, Zhou Z, Gao J, Zakarija A, McMahon BJ, Stein BL: Bleeding complications in BCR-ABL negative myeloproliferative neoplasms: prevalence, type, and risk factors in a single-center cohort. Int J Hematol. 2015, 102:587-93. 10.1007/s12185-015-1871-4

8. Guglielmelli P, Vannucchi AM: Current management strategies for polycythemia vera and essential thrombocythemia. Blood Rev. 2020, 42:100714. 10.1016/j.blre.2020.100714

9. Tiede A, Rand JH, Budde U, Ganser A, Federici AB: How I treat the acquired von Willebrand syndrome. Blood. 2011, 117:6777-85. 10.1182/blood-2010-11-297580

10. Mohri H, Motomura S, Kanamori H, et al.: Clinical significance of inhibitors in acquired von Willebrand syndrome. Blood. 1998, 91:3623-9. 10.1182/blood.V91.10.3623

11. Federici AB, Rand JH, Bucciarelli P, et al.: Acquired von Willebrand syndrome: data from an international registry. Thromb Haemost. 2000, 84:345-9.

12. Kutti J, Weinfeld A: Platelet production and platelet survival in polycythaemia vera with special reference to the spleen size. Scand J Haematol. 1972, 9:97-105. 10.1111/j.1600-0609.1972.tb00917.x

13. Teng CM, Ko FN, Wang JP, Lin CN, Wu TS, Chen CC, Huang TF: Antihaemostatic and antithrombotic effect of some antiplatelet agents isolated from Chinese herbs. J Pharm Pharmacol. 1991, 43:667-9.

10.1111/j.2042-7158.1991.tb03561.x 